\title{
Produto Funcional de Grafos: Um Modelo para Conexão de Sistemas Multiagentes
}

\author{
A.R.G. LOZANO ${ }^{1}$, A.S. SIQUEIRA ${ }^{2}$, S.R.P. MATTOS $^{3 *}$ e S. JURKIEWICZ ${ }^{4}$
}

Recebido em 28 março, 2016 / Aceito em 18 outubro, 2016

\begin{abstract}
RESUMO. Neste trabalho, os conceitos de produto funcional de grafos e coloração total equilibrada foram utilizados para propor um modelo de conexão entre sistemas multiagentes. Mostramos como gerar uma família de grafos regulares que admite coloração de vértice com folga $\Delta$ com $\Delta+1$ cores, denominada grafos harmônicos. Esse resultado demonstra que esses grafos dispõem de uma estrutura escalável e recursiva, pois a partir de uma instância básica inicial, pode-se expandir dinamicamente sua forma, mantendo-se propriedades como conexidade e regularidade. Finalizamos apresentando um modelo de conexão entre sistemas multiagentes (SMA), com a utilização dos grafos harmônicos como suporte para construção de topologias de rede overly P2P para a comunicação entre estes sistemas.
\end{abstract}

Palavras-chave: produto funcional de grafos, grafos harmônicos, sistemas multiagentes.

\section{INTRODUÇÃO}

Diversos campos da matemática empregam, de alguma forma, a noção de produto, um conceito que permite a combinação ou a decomposição de suas estruturas elementares. Na teoria de grafos existem vários tipos de produtos, e entre eles, destacamos o produto cartesiano, o produto forte, o produto direto e o produto lexigráfico [5], cada um com seu próprio conjunto de aplicações e interpretação teórica. Neste artigo o assunto central abordado é o produto funcional de grafos, uma generalização do produto cartesiano de grafos, introduzido em 2011 por Lozano, Siqueira \& Jurkiewicz [16] para auxiliar na construção dos grafos harmônicos.

\footnotetext{
*Autor correspondente: Sergio Ricardo Pereira de Mattos.

${ }^{1}$ Departamento de Matemática da FFP/UERJ, Programa de Pós-Graduação em Ensino das Ciências da ECELAH/ UNIGRANRIO, Grupo de Pesquisa em Matemática Discreta e Computacional. E-mail: arglozano@terra.com.br

${ }^{2}$ Programa de Pós-Graduação em Humanidades, Cultura e Artes e Programa de Pós-Graduação em Ensino das Ciências da ECELAH/UNIGRANRIO, Grupo de Pesquisa em Matemática Discreta e Computacional.

E-mail: asiqueira@unigranrio.edu.br

${ }^{3}$ Programa de Doutorado em Engenharia de Produção da COPPE/UFRJ. Escola de Ciências, Educação, Letras, Artes e Humanidades da UNIGRANRIO, Grupo de Pesquisa em Matemática Discreta e Computacional.

E-mail: rickdemattos@ufrj.br

${ }^{4}$ Programa de Engenharia da Produção da COPPE/UFRJ. E-mail: jurki@pep.ufrj.br
} 
Historicamente os grafos produto, mais especificamente, os grafos produto cartesiano foram largamente empregados como topologia de redes de interconexão. Topologias clássicas como, malha, hiper-estrela, estrela-cubo, hipercubo e toro são obtidas através do produto cartesiano de grafos. Atualmente, o conceito de redes de interconexão (estruturas físicas) não tem a mesma relevância de tempos atrás, entretanto, o conceito de sistemas multiagentes (SMA), no qual dois ou mais agentes trabalham em conjunto de forma a desempenhar determinadas tarefas, vem ganhando cada vez mais espaço e aplicabilidade $[6,14]$. É sobre este tripé (produto funcional de grafos, grafos harmônicos e sistemas multiagentes) que este trabalho se apoia.

Entende-se aqui por conexão de sistemas multiagentes a ligação entre dois ou mais sistemas multiagentes, de modo que agentes de um SMA possam comunicar-se com agentes de outro SMA, possibilitando acesso à serviços, compartilhamento de recursos e garantindo o trabalho em conjunto.

Neste artigo provamos que o produto funcional de grafos permite construir grafos harmônicos, a partir de qualquer grafo regular. Mostramos que a família dos grafos harmônicos oferecem vantagens na sua implementação como topologia de rede overly P2P para comunicação entre SMA. Mostramos também que o conceito de coloração total equilibrada pode ser utilizado para elaborar algoritmos paralelos que independem da topologia da rede. Por fim, propomos um modelo de conexão entre SMA, mediante a utilização dos grafos harmônicos como suporte para a construção destas topologias. Assim, as principais contribuições deste trabalho são o teorema 3.4, o colorário 3.1 e a aplicação do produto funcional de grafos na conexão entre SMA.

Este texto está organizado da seguinte forma: na seção 2 apresentamos o conceito de produto funcional de grafos. A seção 3 aborda a construção dos grafos harmônicos. Na seção 4 adentramos no aspecto computacional, discutimos os conceitos de agentes, sistemas multiagentes e as vantagens da implementação de um sistema de comunicação peer-to-peer na comunicação dos agentes de um SMA. Na seção 5 propomos um modelo de conexão entre SMA com a utilização dos grafos harmônicos, e na seção 6, fazemos as considerações finais.

\section{PRODUTO FUNCIONAL DE GRAFOS}

Conceitos introdutórios sobre grafos e coloração podem ser encontrados em [2] e [20]. Conceitos mais específicos sobre coloração, tais como coloração total equilibrada e coloração com folga de ordem $k$, podem ser vistos em [9] e [4], respectivamente. Para as definições de produto cartesiano de grafos, utilizamos [15] e [18]. A seguir, apresentamos o conceito de produto funcional de grafos que também pode ser encontrado em [8] e [16]. Para melhor entendimento desta seção, se fazem necessárias algumas definições e notações iniciais.

\subsection{Definições e notações iniciais}

- $\Delta(G)$ ou $\Delta$ se não houver ambiguidade, denota o grau máximo do grafo $G$;

- $F(X)$ denota o conjunto de todas as bijeções de $X$ em $X$; 
- $D(G)$ denota o digrafo obtido pela substituição de cada aresta $u v$ do grafo $G$ pelos arcos $(u, v)$ e $(v, u)$, mantendo o mesmo conjunto de vértices;

- $\mathcal{D}$ denota o conjunto dos digrafos que satisfazem as seguintes condições:

1. $(u, v)$ é um arco do digrafo se, e somente se, $(v, u)$ também é um arco do digrafo,

2. Não existem dois arcos iguais.

- Se $\vec{G} \in \mathcal{D}, G(\vec{G})$ denota o grafo obtido pela substituição de cada par de arcos $(u, v)$ e $(v, u)$ de $\vec{G}$ pela aresta $u v$, mantendo o mesmo conjunto de vétices;

- $K_{n}$ denota o grafo completo de $n$ vértices.

Definição 2.1. Os digrafos $\vec{G}_{1}\left(V_{1}, E_{1}\right)$ e $\vec{G}_{2}\left(V_{2}, E_{2}\right)$ são ditos funcionalmente ligados pelas aplicações $f_{1}: E_{1} \rightarrow F\left(V_{2}\right)$ e $f_{2}: E_{2} \rightarrow F\left(V_{1}\right)$ se $f_{1}$ e $f_{2}$ são tais que:

1. Para todo arco $(u, v) \in E_{1}$ se $(v, u) \in E_{1}$, então $f_{1}((u, v))=\left(f_{1}((v, u))\right)^{-1}$,

2. Para todo $(x, y) \in E_{2}$ se $(y, x) \in E_{2}$, então $f_{2}((x, y))=\left(f_{2}((y, x))\right)^{-1}$,

3. Para todo par de $\operatorname{arcos}(u, v) \in E_{1} e(x, y) \in E_{2}$, tem-se que $f_{2}((x, y))(u) \neq v$ ou $f_{1}((u, v))(x) \neq y$.

As aplicações $f_{1}$ e $f_{2}$ são denominadas aplicações de ligação.

Definição 2.2. Sejam dois grafos $G_{1}\left(V_{1}, E_{1}\right)$ e $G_{2}\left(V_{2}, E_{2}\right)$. Se $D\left(G_{1}\right)$ e $D\left(G_{2}\right)$ são funcionalmente ligados pelas aplicações $f_{1}: E\left(D\left(G_{1}\right)\right) \rightarrow F\left(V_{2}\right)$ e $f_{2}: E\left(D\left(G_{2}\right)\right) \rightarrow F\left(V_{1}\right)$, então os grafos $G_{1}\left(V_{1}, E_{1}\right)$ e $G_{2}\left(V_{2}, E_{2}\right)$ são ditos funcionalmente ligados pelas mesmas aplicações.

Definição 2.3. Sejam $\vec{G}_{1}\left(V_{1}, E_{1}\right)$ e $\vec{G}_{2}\left(V_{2}, E_{2}\right)$ digrafos funcionalmente ligados pelas aplicações $f_{1}: E_{1} \rightarrow F\left(V_{2}\right)$ e $f_{2}: E_{2} \rightarrow F\left(V_{1}\right)$. O produto funcional do digrafo $\vec{G}_{1}$ pelo digrafo $\vec{G}_{2}$ segundo as aplicações $f_{1}$ e $f_{2}$, denotado por $\left(\vec{G}_{1}, f_{1}\right) \times\left(\vec{G}_{2}, f_{2}\right)$, é o digrafo $\vec{G}^{*}\left(V^{*}, E^{*}\right)$ definido por:

- $V^{*}=V_{1} \times V_{2}$.

- $((u, x),(v, y)) \in E^{*}$ se, e somente se, uma das seguintes condições for verdadeira:

1. $(u, v) \in E_{1}$ e $f_{1}((u, v))(x)=y$

2. $(x, y) \in E_{2}$ e $f_{2}((x, y))(u)=v$.

Definição 2.4. Sejam $G_{1}\left(V_{1}, E_{1}\right)$ e $G_{2}\left(V_{2}, E_{2}\right)$ grafos funcionalmente ligados pelas aplicações $f_{1}: E\left(D\left(G_{1}\right)\right) \rightarrow F\left(V_{2}\right)$ e $f_{2}: E\left(D\left(G_{2}\right)\right) \rightarrow F\left(V_{1}\right)$. O produto funcional do grafo $G_{1}$ pelo 
grafo $G_{2}$, denotado por $\left(G_{1}, f_{1}\right) \times\left(G_{2}, f_{2}\right)$, é o grafo $G\left(\overrightarrow{G^{*}}\left(V^{*}, E^{*}\right)\right)$, sendo $\overrightarrow{G^{*}}\left(V^{*}, E^{*}\right)=$ $\left(D\left(G_{1}\right), f_{1}\right) \times\left(D\left(G_{2}\right), f_{2}\right)$.

As Figuras 1, 2 e 3 apresentam o produto funcional entre dois caminhos $P_{3}$, segundo as aplicações $f_{1}$ e $f_{2}$. Observe que a Figura 1 faz referência as definições 2.1 e 2.2 , enquanto que as Figuras 2 e 3 ilustram as definições 2.3 e 2.4, respectivamente.
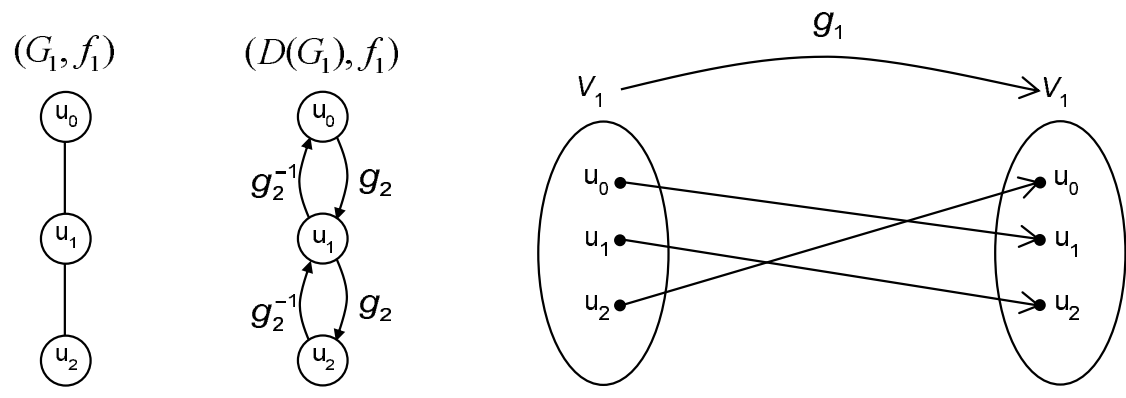

$\left(G_{2}, f_{2}\right) \quad\left(D\left(G_{2}\right), f_{2}\right)$
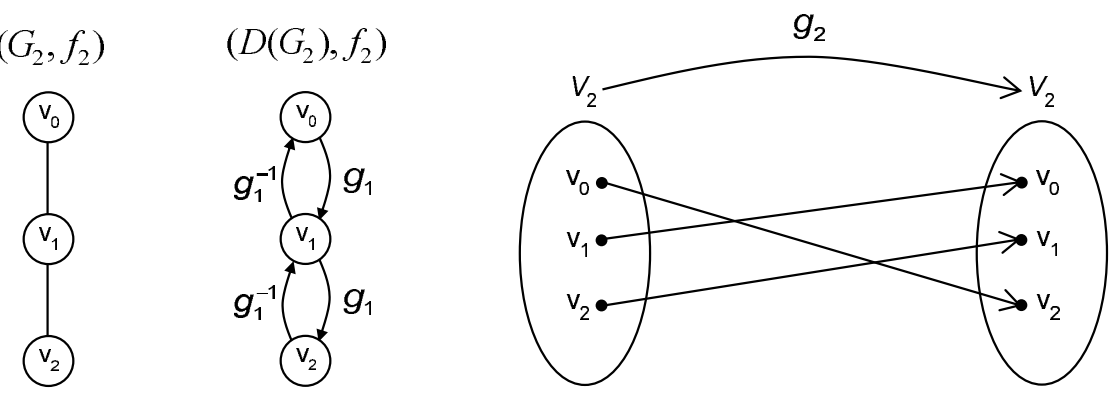

Figura 1: Grafos $G_{1}$ e $G_{2}$, seus respectivos digrafos e as aplicações $f_{1}$ e $f_{2}$.
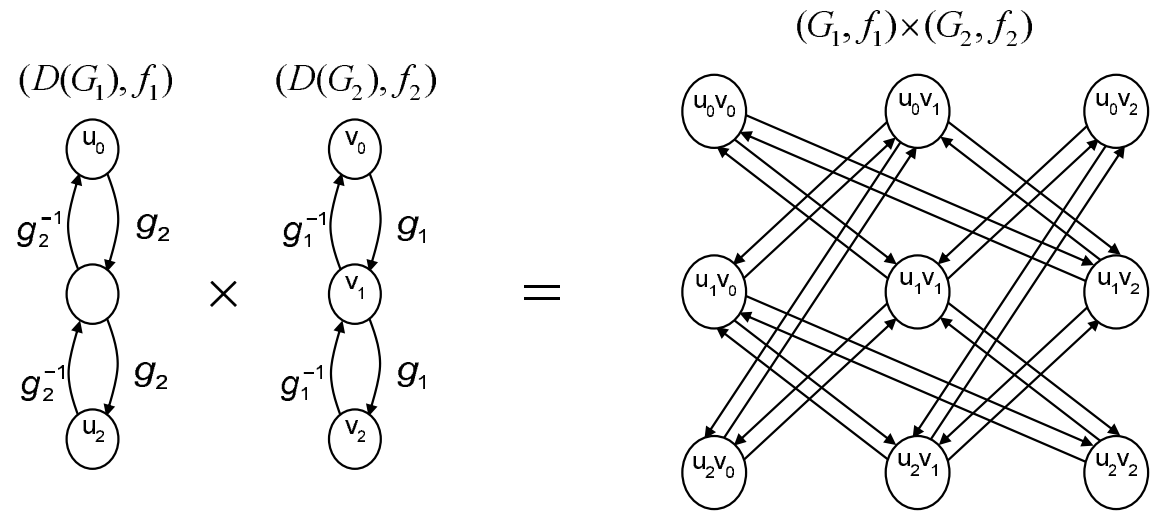

Figura 2: Produto funcional entre os digrafos $D\left(G_{1}\right)$ e $D\left(G_{2}\right)$, segundo $f_{1}$ e $f_{2}$. 


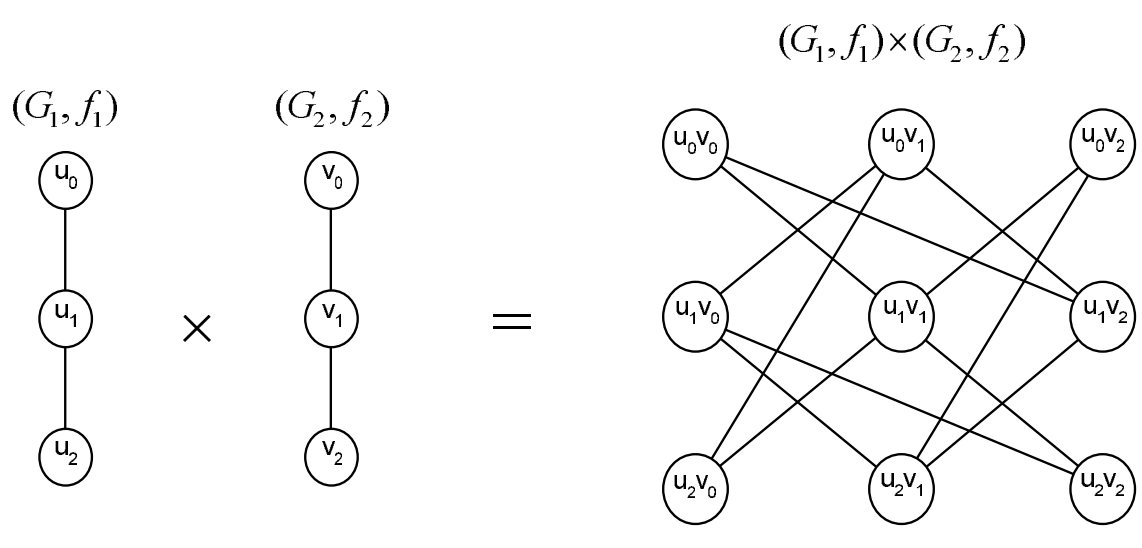

Figura 3: Produto funcional entre os grafos $G_{1}$ e $G_{2}$, segundo $f_{1}$ e $f_{2}$.

\section{GRAFOS HARMÔNICOS}

Nessa seção apresentamos as principais contribuições deste trabalho, o teorema 3.4 e o colorário 3.1 que permitem construir grafos harmônicos, a partir do produto funcional de grafos. Para melhor compreensão desses resultados, enunciamos em 3.1 um teorema de Petersen, descrito em [20] e outros dois resultados apresentados em [4] e [16], respectivamente, que garantem a extensão de uma coloração com folga de ordem $\Delta$ para uma coloração total e equilibrada.

Definição 3.1. Dado um grafo $G(V, E)$, um conjunto de cores $C=\left\{c_{1}, c_{2}, c_{3} \ldots c_{p}\right\}$; com $p \in \mathbb{N}$ e um número natural $k$, tal que $k \leq \Delta(G)$. Uma aplicação $f: V \rightarrow C$ é uma coloração de vértices com folga de ordem $\boldsymbol{k}$ de $G$, se para todo $v \in V$ tem-se que $d(v)<k$, então $|c(N(v))|=d(v)$, caso contrário, $|c(N(v))| \geq k$, sendo $|c(N(v))|$ a cardinalidade do conjunto de cores da vizinhança de $v$ [4].

Definição 3.2. Um grafo $G(V, E)$ regular é dito harmônico, se admite uma coloração de vértices com folga $\Delta$ com $\Delta+1$ cores [16].

Teorema 3.1. Se $G(V, E)$ é um grafo $2 k$-regular então $G$ é 2-fatorizavel [20].

Teorema 3.2. Sejam $G(V, E)$ um grafo e $c: V \rightarrow C=\{1,2,3, \ldots, k\}, k \in N, k \geq \Delta+1$, uma coloração com folga $\Delta$ dos vértices de G. Então, existe uma coloração total de $G$ com no máximo $k+1$ cores [4].

Teorema 3.3. Sejam $G(V, E)$ um grafo regular e $c: V \rightarrow C=\{1,2,3, \ldots, \Delta+1\}$ uma coloração com folga $\Delta$ dos vértices de $G$. Então, a extensão natural de c a $G$, é uma coloração total equilibrada [16].

A coloração obtida pela técnica utilizada na prova do teorema 3.2 é denominada extensão natural de uma coloração com folga de ordem $\Delta$ [16]. 
Teorema 3.4. Para todo grafo regular $G$ e seu complemento $G^{*}$ existem aplicações de ligação $f_{1}$ e $f_{2}$, tais que $\left(G, f_{1}\right) \times\left(G^{*}, f_{2}\right)$ é um grafo harmônico.

Demonstração. Inicialmente observe que para todo grafo regular $G$ tem-se que $\Delta(G)$ ou $\Delta\left(G^{*}\right)$ é par. De fato, se $\Delta(G)$ é ímpar, então $n=|V(G)|$ é par, logo $\Delta\left(K_{n}\right)$ é ímpar e como $\Delta\left(K_{n}\right)=\Delta(G)+\Delta\left(G^{*}\right)$, então $\Delta\left(G^{*}\right)$ é par. Suponhamos inicialmente que $\Delta\left(G^{*}\right)$ é par. Pelo teorema 3.1 existe uma decomposição em 2-fatores de $G^{*}$ e cada 2-fator têm associada uma bijeção. Para todo 2-fator $F$ substituímos cada ciclo por um ciclo orientado e definimos a aplicação $b: V(F) \rightarrow V(F)$, tal que se $(u, v) \in E(F)$, então $b(u)=v$. Claramente $b$ é uma bijeção. A aplicação $f_{2}$ associa a bijeção $b$ a cada arco do ciclo, no ciclo em sentido inverso associamos a bijeção inversa. No grafo $G$ a aplicação $f_{1}$ associa a identidade a todos os pares de arcos associados às arestas. Agora se $V(G)=\left\{v_{1}, v_{2}, \ldots, v_{p}\right\}$ a cada vértice na forma $\left(x, v_{p}\right)$ damos a cor $p$. Por construção, a coloração obtida em $\left(G, f_{1}\right) \times\left(G^{*}, f_{2}\right)$ é uma coloração com folga $\Delta$ com $\Delta+1$ cores. Se $\Delta\left(G^{*}\right)$ é ímpar, então $\Delta(G)$ é par, logo basta trocar as posições de $G$ and $G^{*}$ no raciocínio anterior para obter o resultado desejado. $\operatorname{Logo},\left(G, f_{1}\right) \times\left(G^{*}, f_{2}\right)$ é um grafo harmônico.

Corolário 3.1. Sejam $G$ e $G^{*}$ um grafo regular e seu complemento. Se $\Delta\left(G^{*}\right)$ é par, então para qualquer grafo $G^{\prime}$, tal que $\Delta\left(G^{*}\right)=\Delta\left(G^{\prime}\right)$ existem aplicações de ligação $f_{1}$ e $f_{2}$, tais que $\left(G, f_{1}\right) \times\left(G^{\prime}, f_{2}\right)$ é um grafo harmônico.

Demonstração. Basta observar que tanto $G^{*}$ como $G^{\prime}$ se decompõem na mesma quantidade de 2-fatores, logo o resultado pretendido decorre da mesma argumentação utilizada na prova do teorema 3.4 .

\section{SISTEMAS MULTIAGENTES}

De acordo com Russel \& Norvig [14] "um agente é tudo o que pode ser considerado capaz de perceber seu ambiente por meio de sensores e de agir sobre esse ambientes por intermédio de atuadores". Segundo Lesser [6] "Um SMA é um sistema computacional em que dois ou mais agentes interagem ou trabalham em conjunto, de forma a desempenhar determinadas tarefas ou satisfazer um conjunto de objetivos".

Diante da natureza distribuída dos sistemas multiagentes, Reis [12] destaca como ponto essencial na construção de sociedades de agentes, a capacidade de coordenar as interações e a autonomia nas ações dos diferentes agentes do SMA. O autor também aponta que "para que um agente possa operar como parte do sistema, é necessário a existência de uma infraestrutura que permita a comunicação e/ou interação entre os agentes que compõem o SMA”.

Nesse sentido, Freitas [3] realizou um estudo sobre a comunicação em sistemas multiagentes para tratamento de regiões da Web e apresentou uma série de vantagens na implementação do modelo 
peer-to-peer na comunicação entre os agentes de um SMA. O autor considera que o modelo peer-to-peer é característico de sistemas multiagentes, por ser mais expressivo e abrangente que o modelo cliente-servidor.

Um sistema peer-to-peer implementa uma rede abstrata sobreposta (overlay) em cima da topologia de rede. A rede overlay é uma rede "virtual", e os peers são conectados uns aos outros através de conexões lógicas, na qual todos devem cooperar entre si, disponibilizando parte dos seus recursos em prol da realização de um determinado serviço [1]. O objetivo de um sistema peer-to-peer ( $\mathrm{P} 2 \mathrm{P})$ é compartilhar recursos computacionais através de comunicação direta entre seus componentes, assim qualquer dispositivo pode acessar diretamente os recursos de outros dispositivos do sistema, sem nenhum controle centralizado [1].

Atualmente encontramos diversas topologias implementando redes overlays em sistemas P2P, por exemplo, o Pastry [13] e o Tapestry [19] são baseados na malha; o Chord [17] implementa a topologia de anel, a Edutella [10] utiliza uma arquitetura baseada no hipercubo e o CAN [11] o toro d-dimensional. Pode-se observar que, exceto quanto a topologia em anel, todas as demais são geradas pelo produto cartesiano de grafos, fato que corrobora com a ideia de implementar uma rede overlay em sistemas P2P baseada nos grafos harmônicos.

\section{CONEXÃO ENTRE SISTEMAS MULTIAGENTES}

Nesta seção, propomos um modelo de conexão entre SMA, mediante a utilização dos grafos harmônicos como suporte na construção de topologias de rede overly P2P para comunicação entre estes sistemas.

Os motivos para se ampliar uma estrutura computacional vão desde a abertura de um novo setor de uma empresa à necessidade de compartilhar servidores interligados a internet para suprir a demanda de vendas online durante o lançamento de um produto ou em datas especiais, como no Natal, por exemplo.

No modelo proposto, cada vértice do grafo representa um peer e cada peer representa um agente do SMA, consequentemente, um grafo representa uma rede overlay P2P, que por sua vez representa um sistema multiagente.

Como o produto funcional de dois grafos regulares é um grafo regular [8], tanto o teorema 3.4, quanto o colorário 3.1 podem ser usados reiteradamente para expandir (ou conectar) a topologia inicial, de forma semelhante ao que acontece com o hipercupo, através do produto cartesiano de grafos.

Para ilustrar o processo de construção do grafo harmônico (topologia resultante da conexão dos SMA) destacamos o exemplo apresentado na Figura 4, onde pretende-se conectar três sistemas multiagentes. Como cada SMA é formado por cinco agentes, pode-se utilizar o colorário 3.1, de modo que um grafo $C_{5}$ represente a topologia de cada SMA que se deseja conectar, e um grafo $C_{3}$ caracterize a conexão entre os três sistemas multiagentes. A Figura 5 mostra a contrução do grafo harmônico a partir da aplicação do colorário 3.1 nos grafos $C_{5}$ e $C_{3}$. 


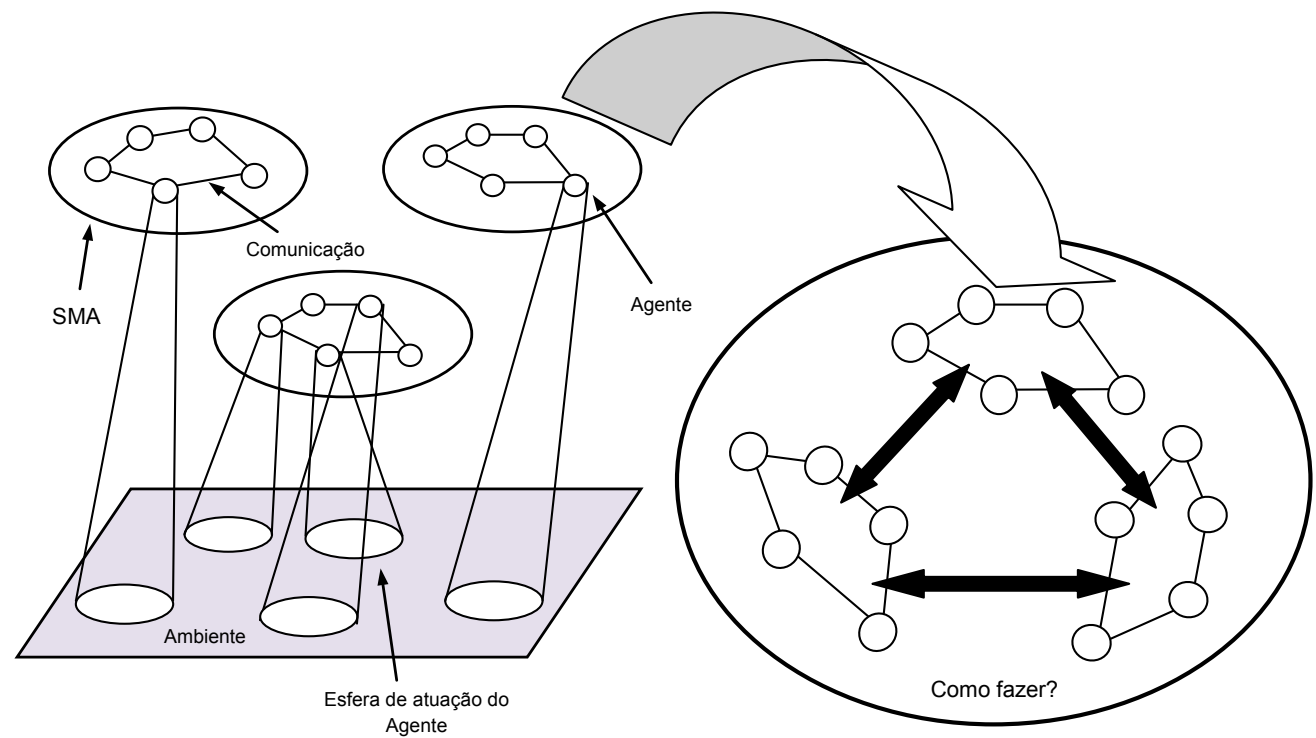

Figura 4: Estrutura de três SMA (esquerda); Conexão entre os três SMA (direita). Adaptado de Reis [12].

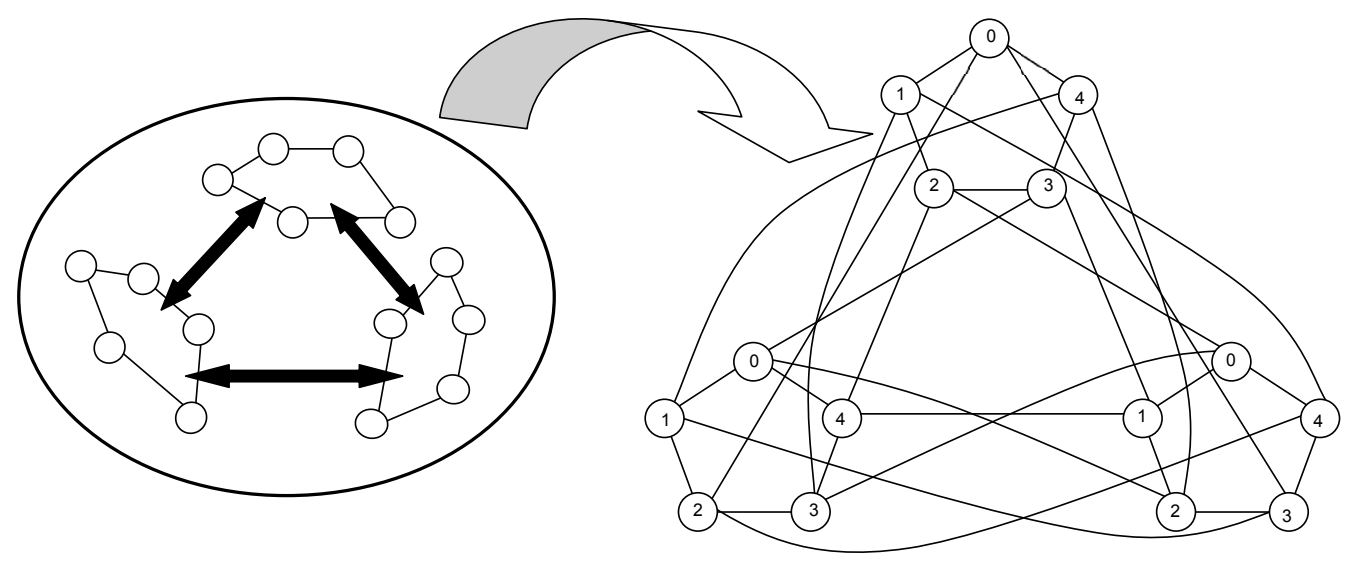

Figura 5: Processo de construção de um grafo harmônico colorido com folga 4 com 5 cores.

Agora basta aplicar os teoremas 3.2 e 3.3 no grafo harmônico resultante para obtermos uma coloração total e equilibrada com no máximo $\Delta+2$ cores. A partir do conceito de coloração total equilibrada é possível oferecer uma representação natural para o processamento paralelo.

\subsection{Algoritmo paralelo de Troca Completa}

Em um algoritmo que envolve troca completa de informações, cada processador possui uma informação e é necessário que todos os processadores conheçam todas as informações. Embora o processamento distribuído tenha vantagens significativas, apresenta como limitações o fato 
da programação geralmente ser feita de forma sequencial e os algoritmos em paralelo serem específicos para cada topologia.

O ideal seria gerar algoritmos comuns a todas as topologias ou paralelizar automaticamente algoritmos sequenciais. Nesse ponto de vista, o algoritmo proposto não é necessariamente ótimo, mas mostra que é possível criar algoritmos que independem da topologia usando a coloração total equilibrada.

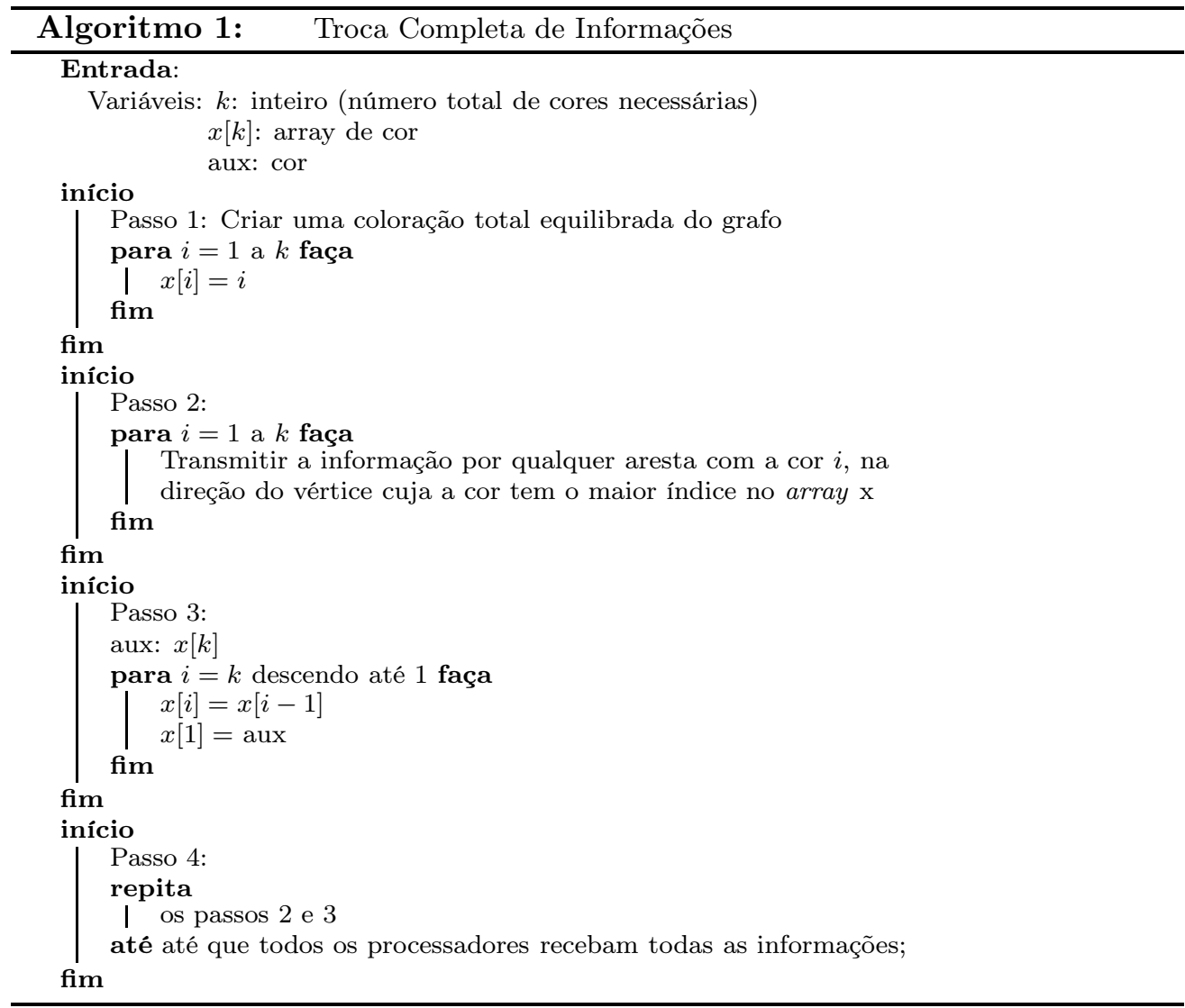

As Figuras 6, 7 e 8 detalham o funcionamento do algoritmo. O grafo corresponde a topologia do SMA, a numeração dos vértices e arestas indicam a coloração total e equilibrada do grafo (passo 1) e as letras acima dos vértices representam as informações contidas nesses vértices (agentes).

Vale ressaltar, que a implementação de topologias que admitem uma coloração total equilibrada pode tornar o processamento mais eficiente, pois permite uma divisão natural dos recursos da rede, onde pelo menos $\frac{t}{\Delta+2}$ processadores ou conexões podem ser usados simultaneamente, sendo $t$ o número de elementos do grafo associado à rede, isto é, número de vértices mais número de arestas e $\Delta$ o grau máximo do grafo. 


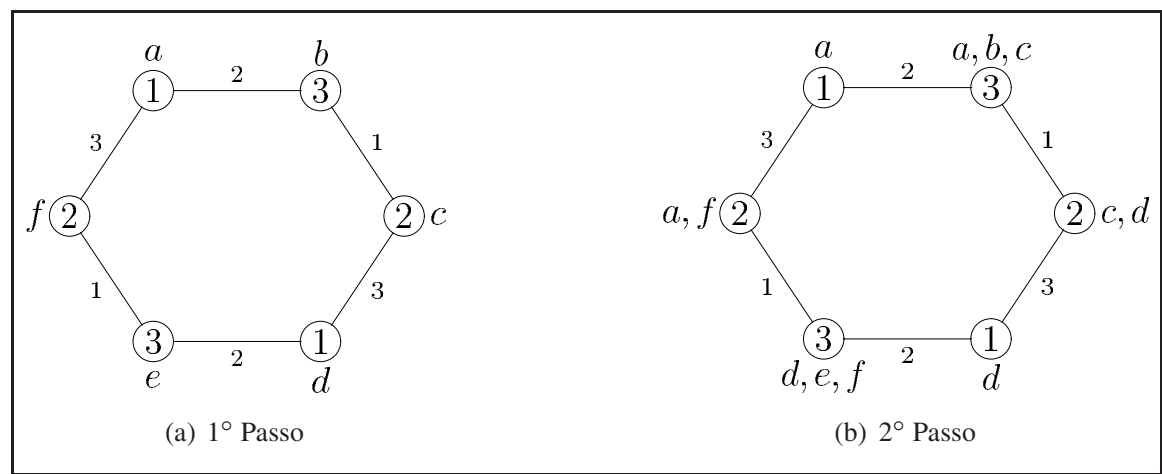

Figura 6: Passo-a-passo do Algoritmo (posição original).

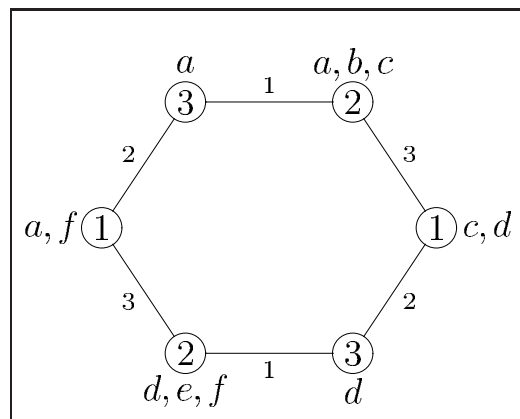

(a) $3^{\circ}$ Passo

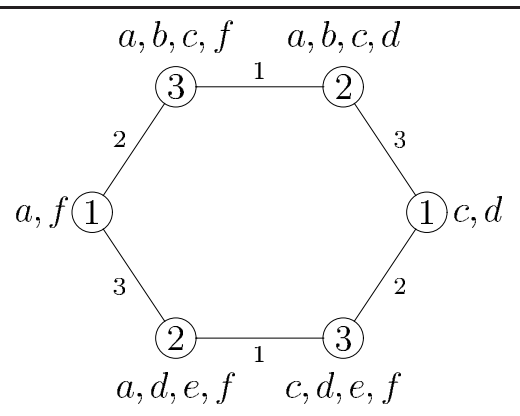

(b) $2^{\circ}$ Passo

Figura 7: Passo-a-passo do Algoritmo ( $1^{a}$ troca).

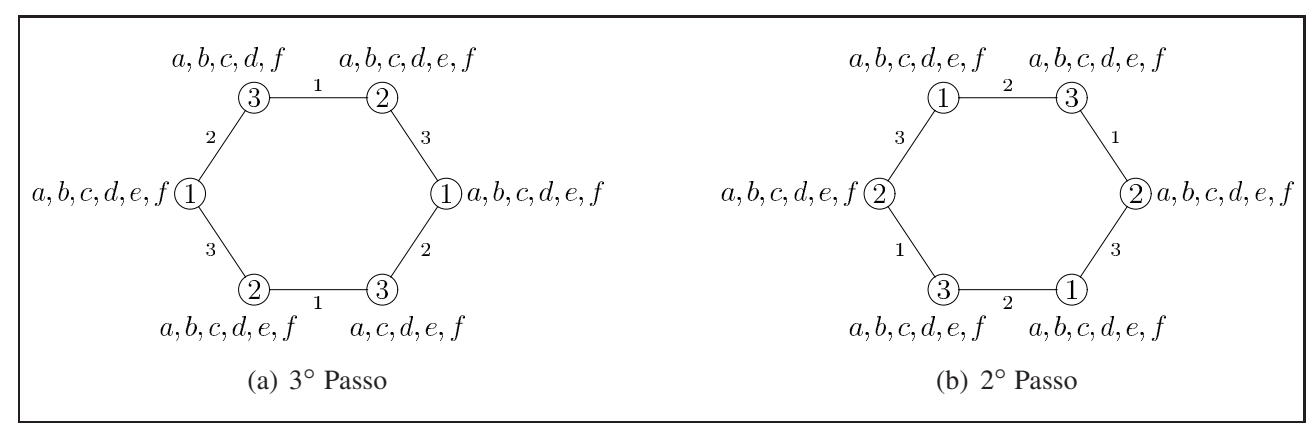

Figura 8: Passo-a-passo do Algoritmo ( $4^{a}$ troca).

Além disso, uma coloração total e equilibrada obtida pela extensão natural de uma coloração com folga de ordem $\Delta$ com $\Delta+1$ cores assegura que para qualquer vértice $v$, todos os vértices vizinhos são coloridos com cores distintas. No caso do grafo ter grau máximo par, a coloração total equilibrada obtida também é feita com $\Delta+1$ cores [16], fato que garante a topologia 
uma otimização no processamento, pois em todo dado momento ou o processador (vértice cor i) está executando uma tarefa ou ele está recebendo informações de um dos seus vizinhos por uma de suas ligações (aresta cor $j$ ), ou seja, em cada instante todo processador do sistema está sendo acionado. Nesse sentido, o uso de algoritmos baseados na coloração total equilibrada não permite a existência de processadores ociosos em nenhum passo de computação.

Não é difícil verificar que topologias como a malha, o hipercubo e o toro nem sempre admitem uma coloração de vértices com folga $\Delta$ com $\Delta+1$ cores. Em contrapartida, dentre as topologias obtidas por meio do produto cartesiano, o hipercubo é a mais escalável e a única que permite um crescimento recursivo conservando sua estrutura original. Sob essa perspectiva, os grafos harmônicos além de serem escaláveis e possuírem uma estrutura recursiva, apresentam como vantagem o fato de admitirem uma coloração total e equilibrada obtida pela extensão natural de uma coloração com folga de ordem $\Delta$ com $\Delta+1$ cores.

\title{
6 CONCLUSÕES
}

Os resultados apresentados neste texto mostram que o produto funcional permite construir uma família de grafos que admitem uma coloração de vértices com folga $\Delta$ com $\Delta+1$ cores. Por outro lado, através do produto funcional pode-se expandir dinamicamente essas estruturas, aumentando ou diminuindo o números de vértices conectados, mantendo propriedades como conexidade e regularidade. Ressaltamos ainda que a partir do conceito de coloração total equilibrada a confecção de algoritmos paralelos pode ser feita de maneira genérica, garantindo uma divisão natural dos recursos de uma rede de conexões. Por fim, mostramos que os grafos harmônicos podem servir como suporte na construção de topologias de rede overly P2P para comunicação entre sistemas multiagentes, bastando para isso optar pela técnica adequada.

\begin{abstract}
In this work, concepts the functional product of graphs and total equitable coloring were used to propose a connection model of multi-agent systems. We show how generate a family of regular graphs that allows vertex coloring handily $\Delta$ with $\Delta+1$ colors, called harmonics graphs. This result shows that these graphs have a scalable and recursive structure because from an initial basic instance, you can dynamically expand their form, keeping properties such as connectivity and regularity. Finally, we present a connection model of between multiagent system (MAS), using the harmonics graphs as support of construction of network topologies overly P2P for communication between these systems.
\end{abstract}

Keywords: functional product of graphs, harmonics graphs, multi-agent systems.

\section{REFERÊNCIAS}

[1] S. Androutsellis-Theotokis \& D. Spinellis. A survey of peer-to-peer content distribution technologies. ACM Computing Surveys (CSUR), 36(4) (2004), 335-371.

[2] J. Bondy \& U. Murty. "Graph Theory with Applications", North-Holland, New York, (1976).

Tend. Mat. Apl. Comput., 17, N. 3 (2016) 
[3] F.L.G. Freitas. "Sistemas Multiagentes Cognitivos para recuperação, classificação e extração integradas de informação da WEB”. Tese de Doutorado, UFSC, Santa Catarina, SC, (2002).

[4] C.V.P. Friedmann, A.R.G. Lozano, L. Markenzon \& C.F.E.M. Waga. Total coloring of Block-cactus graphs. The journal of combinatorial mathematics and combinatorial computing, 78 (2011), 273283.

[5] W. Imrich, S. Klavzar \& D. Rall. "Topics in Graph Theory: Graphs and Their Cartesian Products". AK Peters Ltd, (2008).

[6] V.R. Lesser. Cooperative multiagent systems: A personal view of the state of the art. Knowledge and Data Engineering, IEEE Transactions on, 11(1) (1999), 133-142.

[7] A.R.G. Lozano, C.V.P. Friedmann \& S. Jurkiewicz. Coloração total equilibrada de grafos, um modelo para redes de interconexão. Pesquisa Operacional, 28 (2008), 161-171.

[8] A.R.G. Lozano, A.S. Siqueira, S. Jurkiewicz \& C.V.P. Friedmann. Produto Funcional de Grafos. Tendências em Matemática Aplicada e Computacional, 14 (2013), 221-232.

[9] A.R.G. Lozano. "Coloração Total Equilibrada de Grafos". Tese de Doutorado, COPPE, UFRJ, Rio de Janeiro, RJ, (2005).

[10] W. Nejdl, B. Wolf, C. Qu, S. Decker, M. Sintek, A. Naeve, M. Nilsson, M. Palmer \& T. Risch. EDUTELLA: a P2P Networking Infrastructure based on RDF. Proceedings of the 11th International Conference on World Wide Web, Hawaii, USA, (2002), 604-615.

[11] S. Ratnasamy, P. Francis, M. Handley, R. Karp \& S. Shenker. "A scalable content-addressable network", ACM, (2001).

[12] L.P. Reis. "Coordination in Multi-Agent Systems: Applications in University Management and Robotic Soccer", Ph.D. Thesis, FEUP, Porto, Portugal, (2003).

[13] A. Rowstron \& P. Druschel. Pastry: Scalable, decentralized object location, and routing for large-scale peer-to-peer systems. Middleware 2001, Springer, (2001), 329-350.

[14] S. Russel \& P. Norvig. "Inteligência artificial: A Modern Approach". Prentice Hall Series in Artificial Intelligence, New Jersey, (2004).

[15] G. Sabidussi. Graph multiplication. Math. Z., 72 (1960), 446-457.

[16] A.S. Siqueira. "Coloração total equilibrada em subfamílias de grafos regulares". Tese de Doutorado, COPPE, UFRJ, Rio de Janeiro, RJ, (2011).

[17] I. Stoica, R. Morris, D. Karger, M.F. Kaashoek \& H. Balakrishnan. Chord: A scalable peer-topeer lookup service for internet applications. ACM SIGCOMM Computer Communication Review, 31(4) (2001), 149-160.

[18] V.G. Vizing. The Cartesian product of graphs. Vyc. Sis., 9 (1963), 30-43.

[19] B.Y. Zhao, L. Huang, J. Stribling, S.C. Rhea, A.D. Joseph \& J.D. Kubiatowicz. Tapestry: A resilient global-scale overlay for service deployment. Selected Areas in Communications, IEEE Journal on, 22(1) (2004), 41-53.

[20] H. Yap. “Total colorings of graphs”, Springer, Berlin, (1996). 